\title{
On the Prime Geodesic Theorem for Non-Compact Riemann Surfaces
}

\author{
Muharem Avdispahić, Dženan Gušić \\ Department of Mathematics, Faculty of Sciences and Mathematics, University of Sarajevo, Sarajevo, Bosnia and Herzegovina \\ Email:mavdispa@pmf.unsa.ba,dzenang@pmf.unsa.ba
}

How to cite this paper: Avdispahić, M. and Gušić, Dž. (2016) On the Prime Geodesic Theorem for Non-Compact Riemann Surfaces. Advances in Pure Mathematics, 6, 903914.

http://dx.doi.org/10.4236/apm.2016.612068

Received: October 23, 2016

Accepted: November 19, 2016

Published: November 22, 2016

Copyright $\odot 2016$ by authors and Scientific Research Publishing Inc. This work is licensed under the Creative Commons Attribution International License (CC BY 4.0).

http://creativecommons.org/licenses/by/4.0/

\begin{abstract}
We use B. Randol's method to improve the error term in the prime geodesic theorem for a noncompact Riemann surface having at least one cusp. The case considered is a general one, corresponding to a Fuchsian group of the first kind and a multiplier system with a weight on it.
\end{abstract}

\section{Keywords}

Selberg Trace Formula, Selberg Zeta Function, Prime Geodesic Theorem

\section{Introduction}

The Selberg trace formula, introduced by A. Selberg in 1956, describes the spectrum of the hyperbolic Laplacian in terms of geometric data involving the lengths of geodesics on a Riemann surface. Motivated by analogy between this trace formula and the explicit formulas of number theory relating the zeroes of the Riemann zeta function to prime numbers, Selberg [1] introduced a zeta function whose analytic properties are encoded in the Selberg trace formula. By focusing on the Selberg zeta function, H. Huber ([2], p. 386; [3], p. 464), proved an analogue of the prime number theorem for compact Riemann surfaces with the error term $O\left(x^{\frac{3}{4}}(\log x)^{-\frac{1}{2}}\right)$ that agrees with Selberg's one. Using basically the same method as in [4], D. Hejhal ([5], p. 475), established also the prime geodesic theorem for non-compact Riemann surfaces with the remainder $O\left(x^{\frac{3}{4}}(\log x)^{-\frac{1}{2}}\right)$. However, in the compact case there exist several different proofs (see, B. Randol [6], p. 245; P. Buser [7], p. 257, Th. 9.6.1; M. Avdispahić and L. Smajlović 
[8], Th. 3.1) that give the remainder $O\left(x^{\frac{3}{4}}(\log x)^{-1}\right)$. Thanks to new integral representations of the logarithmic derivative of the Selberg zeta function (cf. [9], p. 185; [10], p. 128), M. Avdispahić and L. Smajlović ([11], p. 13) were in position to improve $O\left(x^{\frac{3}{4}}(\log x)^{-\frac{1}{2}}\right)$ error term in a non-compact, finite volume case up to $O\left(x^{\frac{3}{4}}(\log x)^{-1}\right)$. Whereas the authors in [8] and [11] approached the prime number theorem in various settings via explicit formulas for the Jorgenson-Lang fundamental class of functions, our main goal is to obtain this improvement for non-compact Riemann surfaces with cusps following a more direct method of B. Randol [6].

\section{Preliminaries}

Let $X$ be a non-compact Riemann surface regarded as a quotient $\Gamma \backslash \mathcal{H}$ of the upper half-plane $\mathcal{H}$ by a finitely-generated Fuchsian group $\Gamma \subseteq \operatorname{PSL}(2, \mathbb{R})$ of the first kind, containing $n_{1} \geq 1$ cusps. Let $\mathfrak{I}$ denote the fundamental region of $\Gamma$. We shall assume that the fundamental region $\mathfrak{I}$ of $\Gamma$ has a finite non-Euclidean area $|\mathfrak{I}|$. We put

$$
\bar{\Gamma}=\left\{\left(\begin{array}{ll}
a & b \\
c & d
\end{array}\right) \in S L(2, \mathbb{R}): \frac{a z+b}{c Z+d} \in \Gamma\right\}
$$

and denote by $v$ the multiplier system of the weight $m \in \mathbb{R}$ for $\bar{\Gamma}$. Let $\psi$ be an irreducible $r \times r$ unitary representation on $\Gamma$ and $W(T)=\psi(T) v(T), T \in \bar{\Gamma}$. For an $r$ dimensional vector space $V$ over $\mathbb{C}$ we consider an essentially self-adjoint operator

$$
\Delta_{m}=y^{2}\left(\frac{\partial}{\partial x^{2}}+\frac{\partial}{\partial y^{2}}\right)-i m y \frac{\partial}{\partial x}
$$

on the space $\mathcal{D}_{m}$ of all twice continuously differentiable functions $f: \mathcal{H} \rightarrow V$, such that $f$ and $\Delta_{m}(f)$ are square integrable on $\mathfrak{I}$, and satisfy the equality

$$
f(S z)=\frac{(c z+d)^{m}}{|c z+d|^{m}} W(S) f(z) \text {, for all } z \in \mathcal{H} \text { and } \mathcal{S}=\left(\begin{array}{ll}
a & b \\
c & d
\end{array}\right) \in \bar{\Gamma} \text {. }
$$

The operator $-\Delta_{m}$ has the unique self-adjoint extension $-\tilde{\Delta}_{m}$ to the space $\tilde{\mathcal{D}}_{m}$, a dense subspace of $L^{2}(\Gamma \backslash \mathcal{H})$. Let $T_{j}, \quad j=1, \cdots, n_{1}$ be the set of parabolic transformations corresponding to $n_{1}$ cusps of $\Gamma . W\left(T_{j}\right)$ does not depend on the choice of a representative of the parabolic class $\left\{T_{j}\right\}$ and can be considered as a matrix from $\mathbb{C}^{r \times r}$. By $m_{j}$ we will denote the multiplicity of 1 as an eigen-value of the matrix $W\left(T_{j}\right)$, and $n_{1}^{*}=\sum_{j=1}^{n_{1}} m_{j}$ will be the degree of singularity of $W$. We mention that operator $-\tilde{\Delta}_{m}$ has both the discrete and continuous spectrum in the case $n_{1}^{*} \geq 1$, and only the discrete spectrum in the case $n_{1}^{*}=0$. The discrete spectrum will be denoted as $\left\{\lambda_{n}\right\}_{n>0}\left(0=\lambda_{0}<\lambda_{1}<\cdots<\lambda_{n} \rightarrow \infty\right)$. The continuous spectrum is expressed through 
zeros (or equivalently poles) of the hyperbolic scattering determinant (see, [12]).

\section{Selberg Zeta Function}

Let $\mathrm{P} \Gamma_{\mathrm{h}}$ denotes the set of $\Gamma$-conjugacy classes of a primitive hyperbolic element $P_{0}$ in $\Gamma$, and $\Gamma_{\mathrm{h}}$ denotes the set of $\Gamma$-conjugacy classes of a hyperbolic element $P$ in $\Gamma$ that satisfy property $\operatorname{Tr}(P)>2$. Assume that $|m| \leq 1$. We define the Selberg zeta function associated to the pair $(\Gamma, W)$ by

$$
Z_{\Gamma, W}(s)=\prod_{P_{0} \in \mathrm{P} \Gamma_{\mathrm{h}}} \prod_{k=0}^{\infty} \operatorname{det}\left(I_{r}-W\left(P_{0}\right) N\left(P_{0}\right)^{-s-k}\right) .
$$

$Z_{\Gamma, W}(s)$ is absolutely convergent for $\operatorname{Re}(s)>1$. Analytic considerations given in ([5], pp. 499-501) yield that the Selberg zeta function in this setting satisfies the functional equation

$$
Z_{\Gamma, W}(s) \Psi(s)=Z_{\Gamma, W}(1-s)
$$

with the fudge factor

$$
\Psi(s)=\phi(s) \cdot \eta\left(\frac{1}{2}\right)\left(\int_{\frac{1}{2}}^{s} \frac{\eta^{\prime}}{\eta}(u) \mathrm{d} u\right) .
$$

Here, $\phi$ denotes the hyperbolic scattering determinant. It can be represented in the form

$$
\phi(s)=\left(\frac{\sqrt{\pi} \Gamma(s) \Gamma\left(s-\frac{1}{2}\right)}{\Gamma\left(s+\frac{m}{2}\right) \Gamma\left(s-\frac{m}{2}\right)}\right)^{n_{1}^{*}} \sum_{n=1}^{\infty} \frac{a_{n}}{g_{n}^{2 s}},
$$

where the coefficients $a_{n}$ and $g_{n}$ depend on the group $\Gamma$ (see, [5], p. 437). Here, $n_{1}^{*}$ denotes the degree of singularity of $W$ (see Section 2). An explicit expression for the fudge factor $\eta$ in the Equation (1) is given in ([5], p. 501, Equation (5.10)).

The logarithmic derivative of the Selberg zeta function $Z_{\Gamma, W}(s)$ is given by

$$
\frac{Z_{\Gamma, W}^{\prime}(s)}{Z_{\Gamma, W}(s)}=\sum_{P \in \Gamma_{\mathrm{h}}} \frac{\Lambda(P)}{N(P)^{s}} \operatorname{Tr}(W(P)),
$$

where $N(P)$ denotes the norm of the class $P$ and $\Lambda(P)=\frac{\log N\left(P_{0}\right)}{1-N(P)^{-1}}$ for a primitive element $P_{0}$ such that $P=P_{0}^{n}$ for some $n \in \mathbb{N}$. We will omit the indices in $Z_{\Gamma, W}$ in the sequel.

\section{Counting Functions $\psi_{n}(x, W)$}

Lemma 1. For $\operatorname{Re}(s)>1$,

$$
\frac{Z^{\prime}(s)}{Z(s)}=\sum_{P \in \Gamma_{\mathrm{h}}} \Lambda_{1}(P) \operatorname{Tr}(W(P)) N(P)^{-s}+\frac{Z^{\prime}(s+1)}{Z(s+1)},
$$


where $\Lambda_{1}(P)=\log N\left(P_{0}\right)$ for a primitive element $P_{0}$ such that $P=P_{0}^{n}$ for some $n \in \mathbb{N}$.

Proof.

$$
\begin{aligned}
& \frac{Z^{\prime}(s)}{Z(s)}=\sum_{P \in \Gamma_{\mathrm{h}}} \Lambda_{1}(P) \operatorname{Tr}(W(P))\left(1-N(P)^{-1}\right)^{-1} N(P)^{-s} \\
& =\sum_{P \in \Gamma_{\mathrm{h}}} \Lambda_{1}(P) \operatorname{Tr}(W(P)) N(P)^{-s}+\sum_{P \in \Gamma_{\mathrm{h}}} \Lambda_{1}(P) \operatorname{Tr}(W(P))(N(P)-1)^{-1} N(P)^{-s} \\
& =\sum_{P \in \Gamma_{\mathrm{h}}} \Lambda_{1}(P) \operatorname{Tr}(W(P)) N(P)^{-s}+\sum_{P \in \Gamma_{\mathrm{h}}} \Lambda_{1}(P) \operatorname{Tr}(W(P))\left(1-N(P)^{-1}\right)^{-1} N(P)^{-(s+1)} .
\end{aligned}
$$

We shall spend the rest of this section to derive a representation of $\psi_{2}(x, W)$ in the form (11) bellow. We choose not to write it in a separate statement because of the length of expressions involved. However, it will serve as a base for the proof of the prime geodesic theorem in Section 5.

Let us recall the following theorem given in ([13], p. 51, Th. 40).

Theorem 1. If the Dirichlet's series $f(s)=\Sigma a_{n} e^{-\lambda_{n}}=\Sigma a_{n} l_{n}^{-s}$ is summable $(l, k)$ for $s=\beta$ and $c>0, c>\beta$, then

$$
\omega^{-k} \sum_{l_{n}<\omega} a_{n}\left(\omega-l_{n}\right)^{k}=\frac{1}{2 \pi i} \int_{c-i \infty}^{c+i \infty} f(s) \frac{\Gamma(k+1) \Gamma(s)}{\Gamma(k+1+s)} \omega^{s} \mathrm{~d} s .
$$

By Lemma 1,

$$
\frac{Z^{\prime}(s)}{Z(s)}-\frac{Z^{\prime}(s+1)}{Z(s+1)}=\sum_{P \in \Gamma_{\mathrm{h}}} \Lambda_{1}(P) \operatorname{Tr}(W(P)) N(P)^{-s}
$$

We have,

$$
\begin{aligned}
& \frac{1}{2 \pi i} \int_{c-i \infty}^{c+i \infty}\left(\frac{Z^{\prime}(s)}{Z(s)}-\frac{Z^{\prime}(s+1)}{Z(s+1)}\right) s^{-1}(s+1)^{-1} \cdots(s+k)^{-1} x^{s} \mathrm{~d} s \\
& =\frac{1}{k !} \frac{1}{2 \pi i} \int_{c-i \infty}^{c+i \infty}\left(\sum_{P \in \Gamma_{\mathrm{h}}} \Lambda_{1}(P) \operatorname{Tr}(W(P))\left(\frac{N(P)}{x}\right)^{-s}\right) \frac{\Gamma(k+1) \Gamma(s)}{\Gamma(k+1+s)} \mathrm{d} s
\end{aligned}
$$

Therefore, substituting $\omega=1, f(s)=\sum_{P \in \Gamma_{\mathrm{h}}} \Lambda_{1}(P) \operatorname{Tr}(W(P))\left(\frac{N(P)}{x}\right)^{-s}$, and hence

$$
\begin{aligned}
a_{n}=\Lambda_{1}(P) \operatorname{Tr}(W(P)), l_{n}=\frac{N(P)}{x} \text { in }(2), \text { we get } \\
\qquad \frac{1}{2 \pi i} \int_{c-i \infty}^{c+i \infty}\left(\frac{Z^{\prime}(s)}{Z(s)}-\frac{Z^{\prime}(s+1)}{Z(s+1)}\right) s^{-1}(s+1)^{-1} \cdots(s+k)^{-1} x^{s} \mathrm{~d} s \\
=\frac{1}{k !} \sum_{\frac{N(P)}{x} \leq 1} \Lambda_{1}(P) \operatorname{Tr}(W(P))\left(1-\frac{N(P)}{x}\right)^{-s} \\
=\frac{1}{k !} \sum_{N(P) \leq x} \Lambda_{1}(P) \operatorname{Tr}(W(P))\left(1-\frac{N(P)}{x}\right)^{-s} .
\end{aligned}
$$


Then,

$$
\begin{aligned}
& \frac{1}{2 \pi i} \int_{c-i \infty}^{c+i \infty} \frac{Z^{\prime}(s)}{Z(s)} s^{-1}(s+1)^{-1} \cdots(s+k)^{-1} x^{s} \mathrm{~d} s \\
& =\frac{1}{k !} \sum_{N(P) \leq x} \Lambda_{1}(P) \operatorname{Tr}(W(P))\left(1-\frac{N(P)}{x}\right)^{k} \\
& +\frac{1}{2 \pi i} \int_{c-i \infty}^{c+i \infty} \frac{Z^{\prime}(s+1)}{Z(s+1)} s^{-1}(s+1)^{-1} \cdots(s+k)^{-1} x^{s} \mathrm{~d} s .
\end{aligned}
$$

Now, put

$$
\psi_{0}(x, W)=\sum_{N(P) \leq x} \Lambda_{1}(P) \operatorname{Tr}(W(P))
$$

and

$$
\psi_{j}(x, W)=\int_{0}^{x} \psi_{j-1}(t, W) \mathrm{d} t
$$

for $j=1,2, \cdots$. Using ([14], p. 12, Th. 1.3.5), it is easy to get that

$$
\psi_{j}(x, W)=\frac{1}{j !} \sum_{N(P) \leq x} \Lambda_{1}(P) \operatorname{Tr}(W(P))(x-N(P))^{j} .
$$

For $0<\lambda_{n}<\frac{1}{4}$, let $s_{n}=\frac{1}{2}-i r_{n}=\frac{1}{2}-i \sqrt{\lambda_{n}-\frac{1}{4}}, n=1,2, \cdots, K$, be the zeros of $Z(s)$ in $\left(\frac{1}{2}, 1\right)$. Let $\rho_{k}, k=0,1, \cdots, M_{e}$ denote all zeros of the hyperbolic scattering determinant in $\left(\frac{1}{2}, 1\right]$.

Assume $T>2, T \pm l \neq r_{n}, l \in \mathbb{N}$, where $s_{n}=\frac{1}{2}+i r_{n}$ and $\tilde{s}_{n}=\frac{1}{2}-i r_{r}$ for $r_{n}=-i \sqrt{\frac{1}{4}-\lambda_{n}}, \quad \lambda_{n}>\frac{1}{4}$. Following ([5], p. 468), we may also assume $T \pm l \neq \gamma, \quad l \in \mathbb{N}$, where, $1-\rho, 1-\bar{\rho}$ are the zeros of the Selberg zeta function $Z(s)$ for each zero $\rho=\frac{1}{2}+\eta+i \gamma, \eta \geq 0, \quad \gamma>0$, of the hyperbolic scattering determinant $\phi$. Let $A_{0} \geq \frac{3}{2}$ be a large constant such that $A_{0} \notin \mathbb{Z}, A_{0}+\frac{1}{2} \notin \mathbb{Z}, A_{0} \pm \frac{m}{2} \notin \mathbb{Z}$. We put $A=A_{0}+1$. Without loss of generality we may assume that $c=1+\varepsilon, \varepsilon>0, x \geq 2$. Let $R(A, T)=[-A, c] \times[-T, T]$. By the Cauchy residue theorem one has

$$
\begin{aligned}
& \frac{1}{2 \pi i} \int_{c-i T}^{c+i T} \frac{Z^{\prime}(s)}{Z(s)} s^{-1}(s+1)^{-1} \cdots(s+k)^{-1} x^{s} \mathrm{~d} s \\
& =\frac{1}{2 \pi i}\left(\int_{-A+i T}^{-A_{0}+i T}+\int_{-A_{0}+i T}^{\frac{1}{2}-\varepsilon+i T}+\int_{\frac{1}{2}-\varepsilon+i T}^{\frac{1}{2}+\varepsilon+i T}+\int_{\frac{1}{2}+\varepsilon+i T}^{c+i T}+\int_{c-i T}^{\frac{1}{2}+\varepsilon-i T}+\int_{\frac{1}{2}+\varepsilon-i T}^{\frac{1}{2}-\varepsilon-i T}+\int_{\frac{1}{2}-\varepsilon-i T}^{-A_{0}-i T}+\int_{-A_{0}-i T}^{-A-i T}\right) \\
& +\frac{1}{2 \pi i} \int_{-A-i T}^{-A+i T}+\sum_{z \in R(A, T)} \operatorname{Res}_{s=z}\left(\frac{Z^{\prime}(s)}{Z(s)} s^{-1}(s+1)^{-1} \cdots(s+k)^{-1} x^{s}\right)
\end{aligned}
$$


and

$$
\begin{aligned}
& \frac{1}{2 \pi i} \int_{c-i T}^{c+i T} \frac{Z^{\prime}(s+1)}{Z(s+1)} s^{-1}(s+1)^{-1} \cdots(s+k)^{-1} x^{s} \mathrm{~d} s \\
& =\frac{1}{2 \pi i}\left(\int_{-A+i T}^{-A_{0}+i T}+\int_{-A_{0}+i T}^{\frac{1}{2}-\varepsilon+i T}+\int_{\frac{1}{2}-\varepsilon+i T}^{\frac{1}{2}+\varepsilon+i T}+\int_{\frac{1}{2}+\varepsilon+i T}^{c+i T}+\int_{c-i T}^{\frac{1}{2}+\varepsilon-i T}+\int_{\frac{1}{2}+\varepsilon-i T}^{\frac{1}{2}-\varepsilon-i T}+\int_{\frac{1}{2}-\varepsilon-i T}^{-A_{0}-i T}+\int_{-A_{0}-i T}^{-A-i T}\right) \\
& +\frac{1}{2 \pi i} \int_{-A-i T}^{-A+i T}+\sum_{Z \in R(A, T)} \operatorname{Res}_{s=z}\left(\frac{Z^{\prime}(s+1)}{Z(s+1)} s^{-1}(s+1)^{-1} \cdots(s+k)^{-1} x^{s}\right) .
\end{aligned}
$$

Arguing as in [5] (p. 474) and [4] (pp. 105-108), we easily find that the sum of the first eight integrals on the right hand side of (5) is $O\left(\frac{x^{1+\varepsilon}}{\varepsilon T^{k}}\right)$. Similarly, taking into account that $\frac{Z^{\prime}(s)}{Z(s)}$ is bounded for $\operatorname{Re}(s)>1$, we obtain that the sum of the first eight integrals on the right hand side of (6) is $O\left(\frac{x^{1+\varepsilon}}{\varepsilon T^{k}}\right)$. Following [5] (p. 474) and [4] (p. 85, Prop. 5.7), we obtain that the ninth resp. the third integral on the right hand side of (5) resp. (6) are $O\left(x^{-A}\right)$. Now, if we take $k=2,(5)$ and (6) will give us

$$
\begin{aligned}
& \frac{1}{2 \pi i} \int_{c-i T}^{c+i T} \frac{Z^{\prime}(s)}{Z(s)} \frac{x^{s}}{s(s+1)(s+2)} \mathrm{d} s \\
& =O\left(x^{-A}\right)+O\left(\frac{x^{1+\varepsilon}}{\varepsilon T^{2}}\right)+\sum_{z \in R(A, T)} \operatorname{Res}_{s=z}\left(\frac{Z^{\prime}(s)}{Z(s)} \frac{x^{s}}{s(s+1)(s+2)}\right)
\end{aligned}
$$

and

$$
\begin{aligned}
& \frac{1}{2 \pi i} \int_{c-i T}^{c+i T} \frac{Z^{\prime}(s+1)}{Z(s+1)} \frac{x^{s}}{s(s+1)(s+2)} \mathrm{d} s \\
& =O\left(x^{-A}\right)+O\left(\frac{x^{1+\varepsilon}}{\varepsilon T^{2}}\right)+\sum_{z \in R(A, T)} \operatorname{Res}_{s=z}\left(\frac{Z^{\prime}(s+1)}{Z(s+1)} \frac{x^{s}}{s(s+1)(s+2)}\right) .
\end{aligned}
$$

Bearing in mind location of the poles of $\frac{Z^{\prime}(s)}{Z(s)}$ given in ([5], p. 439, Th. 2.16; or [5], p. 498, Th. 5.3) and the fact that $|m| \leq 1$, we may assume without loss of generality that

$$
-1-\frac{m}{2},-1+\frac{m}{2} \in\left(-\frac{5}{4},-\frac{1}{2}\right],-3-\frac{m}{2},-3+\frac{m}{2} \notin\left[-A,-\frac{5}{2}\right] .
$$

Calculating residues and passing to the limit $T \rightarrow+\infty, A \rightarrow+\infty$ in (7) and (8) we get 


$$
\begin{aligned}
& \frac{1}{2 \pi i} \int_{c-i \infty}^{c+i \infty} \frac{Z^{\prime}(s)}{Z(s)} \frac{x^{s}}{s(s+1)(s+2)} \mathrm{d} s=\frac{1}{6} x+\left(\sum_{n=1}^{K} \frac{x^{s_{n}}}{s_{n}\left(s_{n}+1\right)\left(s_{n}+2\right)}+\sum_{n=1}^{K} \frac{x^{\tilde{s}_{n}}}{\tilde{s}_{n}\left(\tilde{s}_{n}+1\right)\left(\tilde{s}_{n}+2\right)}+\sum_{k=0}^{M_{e}} \frac{x^{1-\rho_{k}}}{\left(1-\rho_{k}\right)\left(2-\rho_{k}\right)\left(3-\rho_{k}\right)}\right. \\
& +A_{0} \frac{x^{-\frac{m}{2}}}{-\frac{m}{2}\left(-\frac{m}{2}+1\right)\left(-\frac{m}{2}+2\right)}+A_{1} \frac{x^{-1-\frac{m}{2}}}{\left(-1-\frac{m}{2}\right)\left(-\frac{m}{2}\right)\left(-\frac{m}{2}+1\right)}+B_{0} \frac{x^{\frac{m}{2}}}{\frac{m}{2}\left(\frac{m}{2}+1\right)\left(\frac{m}{2}+2\right)} \\
& +B_{1} \frac{x^{-1+\frac{m}{2}}}{\left(-1+\frac{m}{2}\right)\left(\frac{m}{2}\right)\left(\frac{m}{2}+1\right)}+\frac{8}{15} \operatorname{Tr}\left(I_{r}-\Phi\left(\frac{1}{2}\right)\right) x^{\frac{1}{2}}-\frac{8}{3} n_{1}^{*} x^{-\frac{1}{2}}-g_{1} n_{1}^{*} x^{-1}-n_{1}^{*} x^{-1} \log x \\
& \left.+\left(-\frac{3}{2}+h_{1}\right) \frac{n_{1}^{*}}{2}+\frac{n_{1}^{*}}{2} \log x\right)+\left(\sum_{r_{n}>0} \frac{x^{s_{n}}}{s_{n}\left(s_{n}+1\right)\left(s_{n}+2\right)}+\sum_{r_{n}>0} \frac{x^{\tilde{s}_{n}}}{\tilde{s}_{n}\left(\tilde{s}_{n}+1\right)\left(\tilde{s}_{n}+2\right)}\right. \\
& \left.+\sum_{\gamma>0} \frac{x^{\frac{1}{2}-\eta-i \gamma}}{\left(\frac{1}{2}-\eta-i \gamma\right)\left(\frac{3}{2}-\eta-i \gamma\right)\left(\frac{5}{2}-\eta-i \gamma\right)}+\sum_{\gamma>0} \frac{x^{\frac{1}{2}-\eta+i \gamma}}{\left(\frac{1}{2}-\eta+i \gamma\right)\left(\frac{3}{2}-\eta+i \gamma\right)\left(\frac{5}{2}-\eta+i \gamma\right)}\right) \\
& +\left(A_{2} \frac{x^{-2-\frac{m}{2}}}{\left(-2-\frac{m}{2}\right)\left(-1-\frac{m}{2}\right)\left(-\frac{m}{2}\right)}+B_{2} \frac{x^{-2+\frac{m}{2}}}{\left(-2+\frac{m}{2}\right)\left(-1+\frac{m}{2}\right) \frac{m}{2}}+\frac{8}{3} n_{1}^{*} x^{-\frac{3}{2}}-\frac{8}{15} n_{1}^{*} x^{-\frac{5}{2}}+\left(\frac{3}{2}+f_{1}\right) \frac{n_{1}^{*}}{2} x^{-2}+\frac{n_{1}^{*}}{2} x^{-2} \log x\right)
\end{aligned}
$$

and

$$
\begin{aligned}
& \frac{1}{2 \pi i} \int_{c-i \infty}^{c+i \infty} \frac{Z^{\prime}(s+1)}{Z(s+1)} \frac{x^{s}}{s(s+1)(s+2)} \mathrm{d} s=\left(\sum_{n=1}^{K} \frac{x^{s_{n}-1}}{\left.s_{n}-1\right) s_{n}\left(s_{n}+1\right)}+\sum_{n=1}^{K} \frac{x^{\tilde{s}_{n}-1}}{\left(\tilde{s}_{n}-1\right) \tilde{s}_{n}\left(\tilde{s}_{n}+1\right)}+A_{0} \frac{x^{-\frac{m}{2}-1}}{\left(-\frac{m}{2}-1\right)\left(-\frac{m}{2}\right)\left(-\frac{m}{2}+1\right)}\right. \\
& +B_{0} \frac{x^{-1+\frac{m}{2}}}{\left(-1+\frac{m}{2}\right) \frac{m}{2}\left(\frac{m}{2}+1\right)}-\frac{8}{3} \operatorname{Tr}\left(I_{r}-\Phi\left(\frac{1}{2}\right)\right) x^{-\frac{1}{2}}+\sum_{k=0}^{M_{e}} \frac{x^{-\rho_{k}}}{\left(-\rho_{k}\right)\left(-\rho_{k}+1\right)\left(-\rho_{k}+2\right)}-g_{1}^{\prime} n_{1}^{*} x^{-1}-n_{1}^{*} x^{-1} \log x \\
& \left.+\left(-\frac{3}{2}+h_{1}^{\prime}\right) \frac{1}{2}+\frac{1}{2} \log x\right)+\left(\sum_{r_{n}>0} \frac{x^{s_{n}-1}}{\left(s_{n}-1\right) s_{n}\left(s_{n}+1\right)}+\sum_{r_{n}>0} \frac{x^{\tilde{s}_{n}-1}}{\left(\tilde{s}_{n}-1\right) \tilde{s}_{n}\left(\tilde{s}_{n}+1\right)}+\sum_{\gamma>0} \frac{x^{-\frac{1}{2}-\eta-i \gamma}}{\left(-\frac{1}{2}-\eta-i \gamma\right)\left(\frac{1}{2}-\eta-i \gamma\right)\left(\frac{3}{2}-\eta-i \gamma\right)}\right. \\
& \left.+\sum_{\gamma>0} \frac{x^{-\frac{1}{2}-\eta+i \gamma}}{\left(-\frac{1}{2}-\eta+i \gamma\right)\left(\frac{1}{2}-\eta+i \gamma\right)\left(\frac{3}{2}-\eta+i \gamma\right)}\right)+\left(A_{1} \frac{x^{-2-\frac{m}{2}}}{\left(-2-\frac{m}{2}\right)\left(-1-\frac{m}{2}\right)\left(-\frac{m}{2}\right)}\right. \\
& \left.+B_{1} \frac{x^{-2+\frac{m}{2}}}{\left(-2+\frac{m}{2}\right)\left(-1+\frac{m}{2}\right) \frac{m}{2}}+\left(\frac{3}{2}+f_{1}^{\prime}\right) \frac{n_{1}^{*}}{2} x^{-2}+\frac{n_{1}^{*}}{2} x^{-2} \log x+\frac{8}{3} n_{1}^{*} x^{-\frac{3}{2}}-\frac{8}{15} n_{1}^{*} x^{-\frac{5}{2}}\right) .
\end{aligned}
$$

The implied constants on the right sides of (9) and (10) depend solely on $\Gamma, m$ and $W$. With $k=2$ in (3), $j=2$ in (4), Equations (4), (3), (9) and (10) yield 


$$
\begin{aligned}
& \psi_{2}(x, W)=\frac{1}{2 !} \sum_{N(P) \leq x} \Lambda_{1}(P) \operatorname{Tr}(W(P))(x-N(P))^{2} \\
& =\frac{1}{2 \pi i}\left(\int_{c-i \infty}^{c+i \infty} \frac{Z^{\prime}(s)}{Z(s)} \frac{x^{s+2}}{s(s+1)(s+2)} \mathrm{d} s-\int_{c-i \infty}^{c+i \infty} \frac{Z^{\prime}(s+1)}{Z(s+1)} \frac{x^{s+2}}{s(s+1)(s+2)} \mathrm{d} s\right) \\
& =\frac{1}{6} x^{3}+\left(\sum_{n=1}^{K} \frac{x^{s_{n}+2}}{s_{n}\left(s_{n}+1\right)\left(s_{n}+2\right)}+\sum_{n=1}^{K} \frac{x^{\tilde{s}_{n}+2}}{\tilde{s}_{n}\left(\tilde{s}_{n}+1\right)\left(\tilde{s}_{n}+2\right)}+\sum_{k=0}^{M_{e}} \frac{x^{3-\rho_{k}}}{\left(1-\rho_{k}\right)\left(2-\rho_{k}\right)\left(3-\rho_{k}\right)}\right. \\
& +A_{0} \frac{x^{-\frac{m}{2}+2}}{-\frac{m}{2}\left(-\frac{m}{2}+1\right)\left(-\frac{m}{2}+2\right)}+A_{1} \frac{x^{1-\frac{m}{2}}}{\left(-1-\frac{m}{2}\right)\left(-\frac{m}{2}\right)\left(-\frac{m}{2}+1\right)}+B_{0} \frac{x^{\frac{m}{2}+2}}{\frac{m}{2}\left(\frac{m}{2}+1\right)\left(\frac{m}{2}+2\right)} \\
& +B_{1} \frac{x^{1+\frac{m}{2}}}{\left(-1+\frac{m}{2}\right)\left(\frac{m}{2}\right)\left(\frac{m}{2}+1\right)}+\frac{8}{15} \operatorname{Tr}\left(I_{r}-\Phi\left(\frac{1}{2}\right)\right) x^{\frac{5}{2}}-\frac{8}{3} n_{1}^{*} x^{\frac{3}{2}}-g_{1} n_{1}^{*} x+\left(-\frac{3}{2}+h_{1}\right) \frac{n_{1}^{*}}{2} x^{2} \\
& +\frac{n_{1}^{*}}{2} x^{2} \log x-\sum_{n=1}^{K} \frac{x^{s_{n}+1}}{\left(s_{n}-1\right) s_{n}\left(s_{n}+1\right)}-\sum_{n=1}^{K} \frac{x^{\tilde{s}_{n}+1}}{\left(\tilde{s}_{n}-1\right) \tilde{s}_{n}\left(\tilde{s}_{n}+1\right)}-A_{0} \frac{x^{-\frac{m}{2}+1}}{\left(-\frac{m}{2}-1\right)\left(-\frac{m}{2}\right)\left(-\frac{m}{2}+1\right)} \\
& -B_{0} \frac{x^{1+\frac{m}{2}}}{\left(-1+\frac{m}{2}\right) \frac{m}{2}\left(\frac{m}{2}+1\right)}+\frac{8}{3} \operatorname{Tr}\left(I_{r}-\Phi\left(\frac{1}{2}\right)\right) x^{\frac{3}{2}}-\frac{1}{2} x^{2} \log x-\sum_{k=0}^{M_{e}} \frac{x^{-\rho_{k}+2}}{\left(-\rho_{k}\right)\left(-\rho_{k}+1\right)\left(-\rho_{k}+2\right)} \\
& \left.+g_{1}^{\prime} n_{1}^{*} x-\left(-\frac{3}{2}+h_{1}^{\prime}\right) \frac{1}{2} x^{2}\right)+\left(\sum_{r_{n}>0} \frac{x^{s_{n}+2}}{s_{n}\left(s_{n}+1\right)\left(s_{n}+2\right)}+\sum_{r_{n}>0} \frac{x^{\tilde{s}_{n}+2}}{\tilde{s}_{n}\left(\tilde{s}_{n}+1\right)\left(\tilde{s}_{n}+2\right)}\right. \\
& +\sum_{\gamma>0} \frac{x^{\frac{5}{2}-\eta-i \gamma}}{\left(\frac{1}{2}-\eta-i \gamma\right)\left(\frac{3}{2}-\eta-i \gamma\right)\left(\frac{5}{2}-\eta-i \gamma\right)}+\sum_{\gamma>0} \frac{x^{\frac{5}{2}-\eta+i \gamma}}{\left(\frac{1}{2}-\eta+i \gamma\right)\left(\frac{3}{2}-\eta+i \gamma\right)\left(\frac{5}{2}-\eta+i \gamma\right)} \\
& -\sum_{r_{n}>0} \frac{x^{s_{n}+1}}{\left(s_{n}-1\right) s_{n}\left(s_{n}+1\right)}-\sum_{r_{n}>0} \frac{x^{\tilde{s}_{n}+1}}{\left(\tilde{s}_{n}-1\right) \tilde{s}_{n}\left(\tilde{s}_{n}+1\right)}-\sum_{\gamma>0} \frac{x^{\frac{3}{2}-\eta-i \gamma}}{\left(-\frac{1}{2}-\eta-i \gamma\right)\left(\frac{1}{2}-\eta-i \gamma\right)\left(\frac{3}{2}-\eta-i \gamma\right)} \\
& \left.-\sum_{\gamma>0} \frac{x^{\frac{3}{2}-\eta+i \gamma}}{\left(-\frac{1}{2}-\eta+i \gamma\right)\left(\frac{1}{2}-\eta+i \gamma\right)\left(\frac{3}{2}-\eta+i \gamma\right)}\right)+\left(A_{2} \frac{x^{-\frac{m}{2}}}{\left(-2-\frac{m}{2}\right)\left(-1-\frac{m}{2}\right)\left(-\frac{m}{2}\right)}\right. \\
& +B_{2} \frac{x^{\frac{m}{2}}}{\left(-2+\frac{m}{2}\right)\left(-1+\frac{m}{2}\right) \frac{m}{2}}+\left(\frac{3}{2}+f_{1}\right) \frac{n_{1}^{*}}{2}+\frac{n_{1}^{*}}{2} \log x-A_{1} \frac{x^{-\frac{m}{2}}}{\left(-2-\frac{m}{2}\right)\left(-1-\frac{m}{2}\right)\left(-\frac{m}{2}\right)} \\
& \left.-B_{1} \frac{x^{\frac{m}{2}}}{\left(-2+\frac{m}{2}\right)\left(-1+\frac{m}{2}\right) \frac{m}{2}}-\left(\frac{3}{2}+f_{1}^{\prime}\right) \frac{n_{1}^{*}}{2}-\frac{n_{1}^{*}}{2} \log x\right) \\
& =\frac{1}{6} x^{3}+\sum_{\mathrm{I}}+\sum_{\mathrm{II}}+\sum_{\mathrm{III}}
\end{aligned}
$$


where the first sum ranges over the finite set of poles $s$ of

$$
\frac{Z^{\prime}(s)}{Z(s)} \frac{x^{s}}{s(s+1)(s+2)}, \frac{Z^{\prime}(s+1)}{Z(s+1)} \frac{x^{s}}{s(s+1)(s+2)}
$$

with $\operatorname{Re}(s)>-\frac{5}{4}, \operatorname{Im}(s)=0$, the second sum ranges over the set of poles $s$ of the same functions with $\operatorname{Im}(s)>0$, and the third sum ranges over the finite set of their poles $s$ with $\operatorname{Re}(s)<-\frac{5}{4}$.

\section{Prime Geodesic Theorem}

In our setting, the prime geodesic counting function is defined by

$$
\pi_{0}(x, W)=\sum_{N\left(P_{0}\right) \leq x} \operatorname{Tr}\left(W\left(P_{0}\right)\right), x \geq 1,
$$

where the sum on the right is taken over all primitive hyperbolic classes $P_{0} \in \mathrm{P} \Gamma_{\mathrm{h}}$ with respect to $\bar{\Gamma}$ (see, [5], p. 473, [11], p. 13).

Theorem 2. For $x \geq 2$, the formula

$$
\pi_{0}(x, W)=\sum_{n=0}^{K} \operatorname{li}\left(x^{s_{n}}\right)+O\left(x^{\frac{3}{4}}(\log x)^{-1}\right)
$$

holds true, where, $s_{n}=\frac{1}{2}+\sqrt{\frac{1}{4}-\lambda_{n}}$ for $0 \leq \lambda_{n}<\frac{1}{4}$, and the implied constant depends solely on $\Gamma, m$ and $W$.

Proof. Following [6] (p. 245) and [15] (p. 11), for a positive number $d>0$, we define the second difference operator $\Delta_{2}^{+}$by

$$
\Delta_{2}^{+} f(x)=\int_{x}^{x+d y+d} \int_{y}^{y+d} f^{\prime \prime}(t) \mathrm{d} t \mathrm{~d} y
$$

Here, $d$ is a constant which will be fixed later. By the mean value theorem, we have

$$
\Delta_{2}^{+} x^{\theta}=d^{2} \theta(\theta-1) \tilde{x}^{\theta-2}
$$

for some $\tilde{x} \in[x, x+2 d]$. It is easy to verify that

$$
\Delta_{2}^{+} f(x)=f(x+2 d)-2 f(x+d)+f(x) .
$$

Reasoning as in [5] (p. 475), we may assume without loss of generality that $\psi_{0}(x, W)$ is non-decreasing. Hence, (12) implies

$$
\psi_{0}(x, W) \leq d^{-2} \Delta_{2}^{+} \psi_{2}(x, W) \leq \psi_{0}(x+2 d, W) .
$$

Since (14) holds true, one can easily deduce that $\Delta_{2}^{+} C f(x)=C \Delta_{2}^{+} f(x), \Delta_{2}^{+} C=0$, $\Delta_{2}^{+} x=0, d^{-2} \Delta_{2}^{+} x^{2}=O(1), d^{-2} \Delta_{2}^{+} x^{2} \log x=O\left(x^{\frac{1}{4}} \log x\right)=O\left(x^{\frac{3}{4}}\right), d^{-2} \Delta_{2}^{+} \log x=O(1)$, $d^{-2} \Delta_{2}^{+}\left(\frac{1}{6} x^{3}\right)=x+O(d)$. Thus, (13) and finiteness of the sums contained in $\sum_{\mathrm{I}}$ on the right hand side (11) yield 


$$
d^{-2} \Delta_{2}^{+}\left(\frac{1}{6} x^{3}+\sum_{\mathrm{I}}\right)=x+O(d)+\sum_{n=1}^{K} \frac{x^{s_{n}}}{S_{n}}+O\left(x^{\frac{3}{4}}\right)
$$

Similarly,

$$
d^{-2} \Delta_{2}^{+} \sum_{\text {III }}=O\left(x^{-\frac{3}{2}}\right)
$$

In order to estimate $d^{-2} \Delta_{2}^{+} \sum_{\text {II }}$, we will first consider

$$
d^{-2} \Delta_{2}^{+} \sum_{r_{n}>0} \frac{x^{s_{n}+2}}{s_{n}\left(s_{n}+1\right)\left(s_{n}+2\right)} .
$$

By (14) it is evident that

$$
d^{-2} \Delta_{2}^{+} \frac{x^{s_{n}+2}}{s_{n}\left(s_{n}+1\right)\left(s_{n}+2\right)}=O\left(d^{-2}\left|s_{n}\right|^{-3} x^{\frac{5}{2}}\right) .
$$

On the other hand, the mean value theorem (13) gives us

$$
d^{-2} \Delta_{2}^{+} \frac{x^{s_{n}+2}}{s_{n}\left(s_{n}+1\right)\left(s_{n}+2\right)}=O\left(\left|s_{n}\right|^{-1} x^{\frac{1}{2}}\right)
$$

Let $N(t)$ be the number of roots of $Z(s)$ on the critical line $\frac{1}{2}+i x$ in the interval $0<x \leq t$. It is known ([5], p. 477, Th. 3.8) that $N(t) \sim \frac{r|\mathfrak{I}|}{4 \pi} t^{2}$. Taking $M>1$ and following ([3], pp. 463-464; [6], p. 246), we use (19) resp. (18) in the sums over $s_{n}$, $\frac{1}{2}<\left|s_{n}\right|<1,1 \leq\left|s_{n}\right|<M \quad$ resp. sum over $s_{n}, \quad\left|s_{n}\right|>M \quad$ (below) to get

$$
\begin{aligned}
& \left|d^{-2} \Delta_{2}^{+} \sum_{r_{n}>0} \frac{x^{s_{n}+2}}{s_{n}\left(s_{n}+1\right)\left(s_{n}+2\right)}\right| \\
& \leq \sum_{\frac{1}{2}<\left|s_{n}\right|<1}\left|d^{-2} \Delta_{2}^{+} \frac{x^{s_{n}+2}}{s_{n}\left(s_{n}+1\right)\left(s_{n}+2\right)}\right|+\sum_{1 \leq s_{n} \mid<M}\left|d^{-2} \Delta_{2}^{+} \frac{x^{s_{n}+2}}{s_{n}\left(s_{n}+1\right)\left(s_{n}+2\right)}\right| \\
& +\sum_{\left|s_{n}\right|>M}\left|d^{-2} \Delta_{2}^{+} \frac{x^{s_{n}+2}}{s_{n}\left(s_{n}+1\right)\left(s_{n}+2\right)}\right| \\
& \leq C_{1} X^{\frac{1}{2}} \sum_{\frac{1}{2}<\left\langle s_{n}\right|<1}\left|s_{n}\right|^{-1}+C_{1} X^{\frac{1}{2}} \sum_{1 \leq\left|s_{n}\right|<M}\left|s_{n}\right|^{-1}+C_{2} d^{-2} x^{\frac{5}{2}} \sum_{\left|s_{n}\right|>M}\left|s_{n}\right|^{-3} \\
& =O\left(x^{\frac{1}{2}}\right)+C_{1} x^{\frac{1}{2}} \int_{1}^{M} t^{-1} \mathrm{~d} N(t)+C_{2} d^{-2} x^{\frac{5}{2}} \int_{M}^{+\infty} t^{-3} \mathrm{~d} N(t) \\
& =O\left(x^{\frac{1}{2}}\right)+O\left(M x^{\frac{1}{2}}\right)+O\left(d^{-2} x^{\frac{5}{2}} M^{-1}\right) \text {. }
\end{aligned}
$$

Thus,

$$
d^{-2} \Delta_{2}^{+} \sum_{r_{n}>0} \frac{x^{s_{n}+2}}{s_{n}\left(s_{n}+1\right)\left(s_{n}+2\right)}=O\left(x^{\frac{1}{2}}\right)+O\left(M x^{\frac{1}{2}}\right)+O\left(d^{-2} x^{\frac{5}{2}} M^{-1}\right) .
$$


Similarly,

$$
d^{-2} \Delta_{2}^{+} \sum_{r_{n}>0} \frac{x^{\tilde{s}_{n}+2}}{\tilde{s}_{n}\left(\tilde{s}_{n}+1\right)\left(\tilde{s}_{n}+2\right)}=O\left(x^{\frac{1}{2}}\right)+O\left(M x^{\frac{1}{2}}\right)+O\left(d^{-2} x^{\frac{5}{2}} M^{-1}\right)
$$

Observe that $\sum_{|\gamma| \leq t} 1=O\left(t^{2}\right)$ (see, [5], p. 437, Prop. 2.13). Thus, application of $d^{-2} \Delta_{2}^{+}$to the third and the fourth sum in $\sum_{\text {II }}$ gives us

$$
O\left(x^{\frac{1}{2}}\right)+O\left(M x^{\frac{1}{2}}\right)+O\left(d^{-2} x^{\frac{5}{2}} M^{-1}\right)
$$

Let us write

$$
\sum_{\mathrm{II}}=\sum_{1}-\sum_{2}
$$

where $\sum_{1}$ denotes the sum of the first four sums in $\sum_{\mathrm{II}}$ and $\sum_{2}$ denotes the sum of the last four sums in $\sum_{\mathrm{II}}$. Now, Equations (11), (16), (17), (20), (21) and (22) give us

$$
\begin{aligned}
d^{-2} \Delta_{2}^{+} \psi_{2}(x, W)= & x+O(d)+\sum_{n=1}^{K} \frac{x^{s_{n}}}{s_{n}}+O\left(x^{\frac{3}{4}}\right)+O\left(M x^{\frac{1}{2}}\right) \\
& +O\left(d^{-2} x^{\frac{5}{2}} M^{-1}\right)-d^{-2} \Delta_{2}^{+} \sum_{2}
\end{aligned}
$$

Putting $M=x^{\frac{1}{4}}, d=x^{\frac{3}{4}}$, the Equation (23) becomes

$$
d^{-2} \Delta_{2}^{+} \psi_{2}(x, W)=x+\sum_{n=1}^{K} \frac{x^{s_{n}}}{s_{n}}+O\left(x^{\frac{3}{4}}\right)-d^{-2} \Delta_{2}^{+} \sum_{2}
$$

Since the left sides of Equations (20), (21) are $O\left(x^{\frac{3}{4}}\right)$ for such choice of $M$ and $d$, we get $d^{-2} \Delta_{2}^{+} \sum_{1}=O\left(x^{\frac{3}{4}}\right)$. Now, it is obvious that $d^{-2} \Delta_{2}^{+} \sum_{1}=O\left(x^{-\frac{1}{4}}\right)$. Finally, Equation (24) gives us

$$
d^{-2} \Delta_{2}^{+} \psi_{2}(x, W)=x+\sum_{n=1}^{K} \frac{x^{s_{n}}}{s_{n}}+O\left(x^{\frac{3}{4}}\right) .
$$

Returning to (15), we conclude that inequality

$$
\psi_{0}(x, W) \leq x+\sum_{n=1}^{K} \frac{x^{s_{n}}}{s_{n}}+O\left(x^{\frac{3}{4}}\right)
$$

holds true. Following ([15], p. 11), we analogously obtain that

$$
x+\sum_{n=1}^{K} \frac{x^{s_{n}}}{s_{n}}+O\left(x^{\frac{3}{4}}\right) \leq \psi_{0}(x, W) .
$$

Hence,

$$
\psi_{0}(x, W)=x+\sum_{n=1}^{K} \frac{x^{s_{n}}}{s_{n}}+O\left(x^{\frac{3}{4}}\right)
$$


Arguing as in [5] (p. 475) and [4] (p. 113), one immediately sees that equality (25) proves the theorem.

\section{Acknowledgements}

We thank the Editor and the referee for their comments.

\section{References}

[1] Selberg, A. (1956) Harmonic Analysis and Discontinuous Groups in Weakly Symmetric Riemannian Spaces with Applications to Dirichlet Series. Journal of the Indian Mathematical Society, 20, 47-87.

[2] Huber, H. (1961) Zur analytischen Theorie hyperbolischer Raumformen und Bewegungsgrupen II. Mathematische Annalen, 142, 385-398. https:/doi.org/10.1007/BF01451031

[3] Huber, H. (1961) Nachtrag zu. Mathematische Annalen, 143, 463-464. https:/doi.org/10.1007/BF01470758

[4] Hejhal, D. (1973) The Selberg Trace Formula for PSL(2, $\mathbb{R})$, Vol. I. Lecture Notes in Mathematics, Volume 548. Springer-Verlag, Berlin-Heidelberg.

[5] Hejhal, D. (1983) The Selberg Trace Formula for PSL(2, $\mathbb{R})$, Vol. II. Lecture Notes in Mathematics, Volume 1001. Springer-Verlag, Berlin-Heidelberg.

[6] Randol, B. (1977) On the Asymptotic Distribution of Closed Geodesics on Compact Riemann Surfaces. Transactions of the American Mathematical Society, 233, 241-247. https:/doi.org/10.1090/S0002-9947-1977-0482582-9

[7] Buser, P. (1992) Geometry and Spectra of Compact Riemann Surfaces, Progress in Mathematics, Vol. 106. Birkhäuser, Boston-Basel-Berlin.

[8] Avdispahić, M. and Smajlović, L. (2009) On the Prime Number Theorem for a Compact Riemmann Surface. Rocky Mountain Journal of Mathematics, 39, 1837-1845. https:/doi.org/10.1216/RMJ-2009-39-6-1837

[9] Avdispahić, M. and Smajlović, L. (2006) An explicit Formula and Its Application to the Selberg Trace Formula. Monatshefte für Mathematik, 147, 183-198. https:/doi.org/10.1007/s00605-005-0317-0

[10] Avdispahić, M. and Smajlović, L. (2008) Euler Constants for a Fuchsian Group of the First Kind. Acta Arithmetica, 131, 125-143. https:/doi.org/10.4064/aa131-2-2

[11] Avdispahić, M. and Smajlović, L. (2016) Selberg Trace Formula as an Explicit Formula and the Prime Geodesic Theorem. (Submitted)

[12] Fischer, J. (19760 An Approach to the Selberg Trace Formula via Selberg Zeta-Function. Lecture Notes in Mathematics, Volume 1253. Springer-Verlag, Berlin-Heidelberg.

[13] Hardy, G.H. and Riesz. M. (1915) The General Theory of Dirichlet's Series. Cambridge University Press, Cambridge.

[14] Jameson, G.J. (2003) The Prime Number Theorem. Cambridge University Press, Cambridge. https:/doi.org/10.1017/CBO9781139164986

[15] Park, J. (2010) Ruelle Zeta Function and Prime Geodesic Theorem for Hyperbolic Manifolds with Cusps. In: van Dijk, G. and Wakayama, M., Eds., Casimir Force, Casimir Operators and Riemann Hypothesis, de Gruyter, Berlin, 89-104. 
Submit or recommend next manuscript to SCIRP and we will provide best service for you:

Accepting pre-submission inquiries through Email, Facebook, LinkedIn, Twitter, etc.

A wide selection of journals (inclusive of 9 subjects, more than 200 journals)

Providing 24-hour high-quality service

User-friendly online submission system

Fair and swift peer-review system

Efficient typesetting and proofreading procedure

Display of the result of downloads and visits, as well as the number of cited articles

Maximum dissemination of your research work

Submit your manuscript at: http://papersubmission.scirp.org/

Or contact apm@scirp.org 